\title{
LA LENGUA DE MARGARITA: EL SILENCIO IMPUESTO Y LA ESCRITURA ACTIVISTA EN CRÓNICA DEL DESAMOR
}

Kristin Kerbavaz

\section{(c) $(7)(\mathrm{F}$}

Esta obra está bajo una licencia Creative Commons 



\title{
LA LENGUA DE MARGARITA: EL SILENCIO IMPUESTO Y LA ESCRITURA ACTIVISTA EN CRÓNICA DEL DESAMOR
}

\author{
MARGARITA'S TONGUE: IMPOSED SILENCE AND ACTIVIST \\ WRITING IN CRÓNICA DEL DESAMOR BY ROSA MONTERO
}

Kristin Kerbavaz

\begin{abstract}
RESUMEN
Esta crítica feminista examina la novela Crónica del desamor por Rosa Montero y su presentación del silenciamiento de la voz femenina en la España de la Transición democrática. En particular, analiza las experiencias de unas personajes de la novela, quienes se encuentran silenciadas en situaciones profesionales, familiares y sexuales. Además, este trabajo considera la escritura femenina dentro de la novela de Montero y propone que esta escritura les presenta una oportunidad de expresarse a las mujeres de la novela y, tras la metaficción, a las mujeres del mundo real también. Palabras clave: Transición democrática, escritura femenina, papeles de género, desencanto político, independencia femenina.
\end{abstract}

\begin{abstract}
This feminist critique examines the novel Cronica del desamor by Rosa Montero and its presentation of the silencing of the female voice in Spain during the Democratic Transition. In particular, it analyzes the experiences of female characters in the novel, who find themselves silenced in professional, familial, and sexual situations. Furthermore, this article considers female authorship within Montero's novel and proposes that this authorship presents an opportunity for self-expression not only to the women in the novel, but also, though metafiction, to the women of the real world.

Keywords: Democratic transition, female authorship, gender roles, political disenchantment, female independence.
\end{abstract}

En la introducción de la edición de 1999 de su novela Crónica del desamor, Rosa Montero admite: "Lo llamé Crónica porque ni siquiera me atrevía a llamarlo novela" (p. 11). Su primera novela, publicada apenas cuatro años después de la muerte del General Francisco Franco, trasciende su existencia como obra de ficción y llega a ser un libro con verdaderas implicaciones sociales. Cuenta las experiencias de unas mujeres españolas en los años setenta

Bach. Kristin Kerbavaz. University of Puget Sound.

Correo electrónico: k.kerbavaz@gmail.com

Recepción: 13- 05- 2015

Aceptación: 27- 05- 2015 
y revela que, aunque les hubieran prometido la liberación, la Transición democrática y la sociedad "nueva" que supuestamente se creó perpetuaban el silenciamiento institucional de la mujer. En Crónica del desamor, entonces, Rosa Montero presenta una sociedad en que se oprime la voz femenina y se pretende remediar esta situación a través de la escritura.

La novela de Montero trata de un entorno social e histórico completamente único. Con la muerte de Franco el 20 de noviembre de 1975, se acabó una dictadura opresiva. Sin embargo, la opresión de la mujer, en particular, no murió con el caudillo:

With [ Franco's ] death, franquismo was revealed to be the giant cover under which machismo had been hiding, and it signaled the beginning of the younger generation's fight against the patriarchy, against a value system that had been camouflaged for so long under the name of franquismo. (Jasper, 2005, p. 117)

El machismo siguió permeando la sociedad española durante la Transición hacia la democracia. Aunque había movimientos de liberación femenina, no pudieron deshacerse por completo de la cultura falocéntrica de España (Davies, 1994, p. 97). Más que nada, la falta de cambios concretos se basa en la misma Transición: se priorizaba el cambio político, y los cambios sociales que necesitaban acompañarlo se cayeron, olvidados, a los márgenes (Marcone, 1998, p. 65). El resultado fue un gran sentido de desencanto; llegó la democracia, pero la injustica continuaba (Escudero, 2006, p. 40). Como la narradora lo describe en la novela,

[...] todas esas mujeres entre treinta y cuarenta años que se saben perdedoras, que han comprendido que el
tren ha salido dejándolas en tierra, todas esas mujeres inteligentes, sensibles, amables, que han renunciado a
vivir porque el cambio les ha llegado demasiado tarde, porque se sienten incapaces. (Montero, 1979, p. 200)

Las mujeres de las que escribe Montero son capaces pero han sido lisiadas por una cultura y unas instituciones que no las valoran. Aunque el cambio democrático - un aspecto de la modernización que el tren representa- ha marchado hacia arriba, las mujeres no están a bordo. El progreso las ha dejado atrás. Crónica del desamor, entonces, las encuentra en la plataforma: expresa su predicamento, su desilusión y sus intentos de cambiar las circunstancias. Es una novela que trata de la mujer española y su lucha por subir al tren del progreso aunque el mundo patriarcal no le da la mano (Escudero, 2006, p. 36).

Una de las maneras más obvias en que el patriarcado español oprimía a la mujer a lo largo de su historia fue el silenciamiento de la voz femenina. Joan L. Brown (1991, p. 13) reflexiona sobre la voz de la mujer en el campo literario español:

Only in the contemporary era-the Franco period and the post-Franco years to date-has there been a sizable contingent of women writers in Spain. This should come as no surprise in a culture whose concept of masculine superiority [el machismo] is so strong as to be known in other languages by its Spanish name.

Como nota Brown, aunque las mujeres españolas empezaron a tomar su lugar en el mundo literario en el siglo XX, seguían siendo una minoría. España, incluso en la época en que escribió Montero, se destacó por la exclusión de la voz femenina del discurso nacional (Bárcenas Bautista, 2007, p. 6).

Crónica del desamor se permea con episodios de este silenciamiento. La protagonista, Ana Antón, una escritora en la redacción de una revista semanal, lo experimenta varias veces en relación con sus obras. Su compañero de trabajo Mateo comenta sobre un editorial que Ana ha escrito sobre el hombre español de la posguerra:

-Ana, mujer, esto está muy bien-dice Mateo con la sonrisa amable y blanda que suele poner cuando piensa regañar o criticar a alguien-, pero es que tenéis todos una tendencia a escribir editoriales que es la hostia... Mira, guapita, ¿por qué no pones más datos, o sea, más reportaje de verdad, y dejas que las conclusiones las saque el lector? (Montero, 1979, p. 81) 
Aunque utiliza la forma neutra "todos," su uso de otras expresiones feminizadas como "mujer" y "guapita" hace obvio que Mateo está haciendo una generalización de género. Las opiniones de Ana, como las de todas las mujeres, no tienen ningún valor para Mateo; quiere que no las exprese, entonces, y que deje que el lector (probablemente masculino) opine en su lugar. Aún más, el propio hijo de Ana comparte este descuido hacia la expresión femenina. Cuando encuentra a su madre escribiendo su novela,

[...] Curro calla un momento mirando las hojas cubiertas de menuda letra, luego se deshace del abrazo, joven, cruel y poderoso, y ya en el suelo, comenta con tajante y sabio tono: "pues es una tontería." (Montero, 1979, p. 153)

Al condenar el trabajo de su madre a ser solo una "tontería", Curro revela la profundidad del machismo español. A la edad de cinco años, ya cree que las palabras de las mujeres no valen nada. Como lo expresa Vanessa Knights (1999, p. 88):

Curro is only a small boy but he speaks for the patriarchy in condemning the female voice to silence. Time and time again women are silenced or fall silent in the company of men in this novel in a wide range of situations [...]

Los hombres de la novela desvaloran sus palabras, y las mujeres pierden la habilidad de expresarse. Esto pasa o a causa de la autocensura, como se ve en una reflexión de la narradora al principio de la novela, "Ana ha aprendido a ser precavida. A las preguntas contesta con preguntas para disminuir los riesgos de la batalla" (Montero, 1979, p. 10), o a causa del silenciamiento aprendido tras años de desacato, como se relata casi al final: "Ana se encuentra tonta y zafia, particularmente enmudecida. Sabe que a veces puede resultar casi brillante y divertida, pero en estos momentos se repliega en sí misma, intimidada, tan bloqueada como en sus guateques quinceañeros" (Montero, 1979, p. 253). Ana es una mujer inteligente y carismática, pero no se expresa: o disimula para protegerse a sí misma, o se queda muda. Ha "aprendido a ser precavida," a autocensurarse, porque se ha internalizado el mensaje silenciador del hombre español: lo que dice ella no les importa.

La imposición del silencio en la mujer se extiende a personajes menos centrales también. Candela, una de las amigas de Ana, se acuerda de la historia de Margarita, una chica que se silencia literalmente en un manicomio: "Margarita, una psicótica de diecinueve años, [...] patentizara su miedo y su protesta de la única forma que podía: amputándose la lengua de raíz con una gillette subrepticia" (Montero, 1979, p. 211). Impotente contra la voluntad de los hombres que le controlan la vida, Margarita definitivamente acata al silenciamiento. Es una acción trágica que se repite de manera menos sangrienta en otras situaciones: por ejemplo, Antonia, la madre de Candela, le cuenta a su hija que lloraba por las noches durante la primera década de su matrimonio infeliz y antes de "acostumbrarse": "Los diez primeros años lloré un poco por las noches, en secreto, o bien en las mañanas, con la casa sola, sentada en una cama a medio hacer. Después me acostumbré" (Montero, 1979, p. 214). La narradora cuenta que "Candela notó [...] con espanto que en la boca abierta de su madre florecía el herido muñón de la lengua de Margarita, y entre las carcajadas de Antonia resbalaban cuajarones de sangre amoratada" (Montero, 1979, p. 214). La mujer española típica, el ama de casa, también ha silenciado a sí misma, aprendiendo a acostumbrarse, a aceptar lo que le pasa aunque llora a solas cada noche. Como nota Candela, la mujer española comparte la experiencia de la Margarita herida: sufre, pero no puede gritar. 
Una de las esferas en que más se nota el silencio femenino en Crónica del desamor es la del consentimiento sexual. En esto tiene parte de la culpa la misma "liberación femenina", un movimiento incompleto que les hace daño a las mujeres de la novela:

[Ana] Pensó en la liberación de la mujer, o mejor dicho, en esa supuesta liberación que a ojos de muchos hombres sólo se concretaba en lo sexual, en tener hembras más dispuestas, en olvidar el odiado condón, el coito interrumpido. Los hombres que inventaron la píldora la ofrecieron como clave mágica de la revolución de la mujer, como si eso fuera suficiente. (Montero, 1979, p. 26)

La revolución sexual y el machismo español inherente forman una combinación tóxica. Los cambios supuestamente dirigidos al mejoramiento de la vida de la mujer realmente rodean la experiencia masculina. Ana nota específicamente que eran hombres los científicos que inventaron la píldora y que lo hicieron solamente para disfrutar de sus consecuencias sexuales. A las propias mujeres les consideran subhumanas, nada más que las "hembras" de cualquier otra especie. No reconocen que hay más implicado en un acto sexual que la posibilidad de encontrarse embarazada. Esta idea tiene sus raíces en un problema central de la percepción de la liberación femenina. Nota John Hooper (1995, p. 129) que existía

\footnotetext{
a tendency to link women's liberation with heterosexual permissiveness. Carmen, as portrayed in Carlos Saura's film version, is a promiscuous, predatory troublemaker. Yet when the film came to be shown on television in Spain, the listing in one of the newspapers that day began: 'Carmen, a liberated woman...'
}

El deseo masculino es un problema central del movimiento español de liberación femenina, y creó un doble estándar sexual que se nota en la novela de Montero: las mujeres tienen que ser sexuales, pero solamente al servicio de los deseos masculinos. Carmen, como propone Hooper, llega a ser problemática no porque es activa sexualmente sino porque pretende cumplir con sus propios deseos.

En la novela, Elena se enfrenta con la misma paradoja sexual. Piensa en su adolescencia, cuando se libera definitivamente: "Abandonó definitivamente el hogar [...] para instalarse provisionalmente en casa de su hermana. Fue entonces cuando escogió la filosofía pura. Y cuando llegó a la decisión de dejar de ser virgen” (Montero, 1979, p. 45). Se libera definitivamente: deja atrás la casa familiar y abre la mente hacia la educación. En este momento de libertad, elige también empezar su vida sexual. Pone énfasis en que esta fue una decisión activa, una expresión de subjetividad, y esto es lo que llega a ser problemático: Miguel Ángel, el muchacho con quien quiere tener relaciones sexuales, la rechaza cuando aprende que es virgen. Una virgen que decide controlar su sexualidad no actúa dentro de los parámetros del deseo masculino, y a Miguel Ángel no le interesa ceder el rol de instigador. Después, entonces, le obliga a Elena darle sexo oral en el coche:

\footnotetext{
Ella no está excitada en absoluto, se encuentra a sí misma vacía, abandonada y sin respuestas. Le toca inhábilmente, con reparo y algo de repugnancia, "chúpamela", dice él muy quedo, en un murmullo, "chúpamela", insiste, Elena duda, le da asco, Miguel Ángel empuja suave pero con firmeza su cabeza, ella opone al principio alguna resistencia pero al fin consiente [...]. (Montero, 1979, p. 51)
}

Miguel Ángel reimpone su subjetividad sobre la de Elena; elige, en vez del sexo mutuamente recíproco, que él recibe sexo oral, una experiencia de placer en que ella no participa. Además, es una acción obligada físicamente: aunque Elena insiste en que "al fin consiente," esta aprobación forzada no es consentimiento verdadero. Miguel Ángel viola a Elena para reclamar su propia subjetividad sexual, y para hacerlo literalmente le tapa la boca. Suprime completamente sus deseos e intereses; la silencia totalmente. 
Elena no es la única víctima de la imposición de una sexualidad masculina torcida. La Pulga, otra amiga de Ana, ha experimentado una violación en que la sociedad española fue cómplice:

[...] la Pulga llegó virgen y asustada a la bendecida cama del hotel. Él la violó sin palabras, dolorosa e inhábilmente, y a la mañana siguiente la Pulga despertó en una almohada mojada en lágrimas sintiendo tirantez y escozor entre las piernas. Es una vaginitis, dijo el médico. Y más tarde, tras las pruebas, tras los análisis, añadió, es una vaginitis psicológica. (Montero, 1979, p. 87)

En la cama "bendecida" del matrimonio, la Iglesia Católica endosa su violación. Están casados; entonces lo que él quiere está aprobado socialmente y tácitamente con la bendición de la Iglesia. Cuando el dolor interno de la Pulga se expresa físicamente, el médico, el portavoz de la razón y lo secular, lo relega al mundo de quejas psicológicas. No lo reconoce como problema real y, por lo tanto, descuenta la validez de su experiencia. Lo secular tal como lo religioso de la sociedad española la rechazan a la Pulga, dejándola impotente y sin ayuda.

La sexualidad masculina impuesta no se expresa solamente en forma de violación. Ana da el ejemplo de los piropos constantes y los toques no deseados que han experimentado todas las mujeres desde muy jóvenes y en diferentes espacios públicos:

[S]e hicieron conocedoras de estos asaltos incruentos y cotidianos. De las manos que pellizcan culos, de los restregones de autobús, del asco al intuir algo duro-pobres de ellas, ignorantes de ereccionescontra tu muslo o tu mano. De esas sombras fugaces-padres de familia numerosa, maridos ejemplares, trabajadores fatigados, sin duda-que se precipitaban sobre ti en mitad de la calle, los ojos brillantes, susurrando palabras desconocidas y brutales, te-lo-voy-a-meter-por-no-sé-dónde, te-voy-a-llenar-deleche, te-cogería-y-te, y ellas, que no sabían nada de eso, se encogían contra la esquina, miraban hacia otro lado amedrentadas, aguantaban la respiración mientras el aliento del hombre rebotaba contra ellas [...]. (Montero, 1979, p. 148)

Parecido a la experiencia de Elena con Miguel Ángel, este tipo de acoso representa la manifestación de una sexualidad que es tan unilateral como abusiva y sesgada. Los hombres de la calle no les piden consentimiento a las jóvenes antes de actuar; solamente son objetos de sus propios deseos masculinos. Como respuesta, las chicas aguantan la respiración, una acción defensiva que físicamente no las deja responder. Es un abuso universal para las mujeres de la novela: "se hicieron conocedoras" todas, y los asaltos son "cotidianos". Además, los perpetradores provienen de todas las esferas sociales, e incluso son hombres a quienes se les debe respeto y que no parecen ser inmorales. Como Margarita, Antonia (la madre de Candela y Elena) y la misma Elena con su boca tapada, quedan mudas.

Una falta de consentimiento sexual incluso se ve en relaciones establecidas o aparentemente consensuales. Más de una vez en la novela las mujeres participan en actos sexuales no queridos sin expresar su oposición porque sus parejas ignoran sus protestas o no dejan que las mujeres las articulen. Esto le ocurre a la misma Elena cuando pelea con Javier:

[...] ya Javier la abraza alentado por el aparente deshielo, 'no, espera, espera, Javier, es que de verdad que así no se arregla nada, espera, hablemos un poco', protesta ella, pero Javier hace bromas y dulzuras ignorando sus palabras y al fin se introduce dentro de ella, Elena le admite con melancolía y sin deseo y apenas le acompaña. (Montero, 1979, p. 110)

Tal como ocurrió con Miguel Ángel, cuando "no está excitada en absoluto" a Elena no le interesa tener relaciones sexuales - y lo expresa claramente. Sin embargo, Javier la penetra. Elena disimula en su recuento: crea un sentido falso de consentimiento al expresar que "le admite", igual que, con Miguel Ángel, "al fin consiente" al sexo oral. No obstante, es una 
violación lo que ella experimenta, aunque no violenta físicamente. Algo parecido le sucede a Ana cuando se encuentra vulnerable en compañía de José María, un amante habitual. Según la narradora, "Ana quisiera marchar sola [...]. Pero los llantos están aún demasiado recientes y no ha habido tiempo para serenar la situación. Está claro que la llevará a casa, que subirá con ella, que querrá amarla" (Montero, 1979, p. 246). Presiente claramente cómo terminará la noche y, aunque quiere irse a casa a solas, sabe que acabará acostándose con él. Ni piensa en cómo evitarlo; en la España que Crónica del desamor describe, el deseo masculino siempre suplanta el femenino. "Querrá amarla”, piensa Ana, y allí esta. Ana intenta explicar la situación:

\begin{abstract}
Es, una vez más, una cuestión de estereotipos. El hombre ha de ser tópicamente potente, la mujer tópicamente insaciable. [...] Y por ello Ana finge [el orgasmo], como fingen también millones de mujeres sin decirlo, sin atreverse a confesárselo las unas a las otras tan prisioneras están de su papel de amantes. (Motnero, 1979, pp. 229-230)
\end{abstract}

Parecido al caso de Carmen en la película epónima de Saura, las expectativas sociales que rodean la sexualidad son problemáticas para las mujeres e incluso para los hombres de la novela. Como la Margarita tan emblemática de la mujer en Crónica del desamor, son "prisioneras" tan silenciadas por su rol de "mujer liberada" que ni siquiera pueden ponerses a los hombres ni hablar con otras mujeres de su experiencia. Sufren a solas.

Crónica del desamor presenta una realidad española en que el patriarcado silencia a la mujer. Al llamarla "crónica", Montero asigna su novela al género de textos que registran sucesos históricos en orden cronológico - un género que ha sido dominado históricamente por hombres, como nota Carmen Bárcenas Bautista (2007, p. 65). La novela, entonces, adopta la autoridad de un libro que no es de ficción: habla de una realidad irrefutable, una realidad de la que no hablan ni siquiera los hombres que normalmente escriben las crónicas. Como afirma Bárcenas Bautista (2007, p. 65), "Montero pretende entonces dar cuenta de la situación social en la que se encontraba la mujer española. Al denominarla una 'crónica' y no una novela, Montero pretende revelar la realidad sobre el machismo en la sociedad de la época”. Ann D. Jasper (2005, p. 118) describe Crónica del desamor como un "attempt to photograph that reality, a reality which appeared new but that in fact had been there before November 20, 1975 and therefore was not new at all”. En referencia al título de la novela, Jasper (2005, p. 118) observa:

Her Crónica del desamor [...] was to be just that: the official notice that the patriarchy had been uncovered; a chronicle in which she was to detail the causes of this "desamor," the distance that separated the two sexes.

Por lo tanto, Crónica tiene un propósito social, un rol de activista. Al llamarla así, Montero declara que su novela revela una realidad de la que no habla la mayoría: el propósito de Crónica del desamor es destapar el silencio de las mujeres.

Montero ataca el silencio impuesto a través de la metaficción, una forma literaria que, según Concha Alborg (1988, p. 71), “[...] puede ser considerada como una modalidad subversiva. Al llamar la atención al artífice de la ficción, se sugiere que también puede ser falso el mundo real en el cual se basa". Además de cuestionar la legitimidad del mundo real, la metaficción difumina las divisiones entre la realidad y el espacio literario creativo: deja que los cambios y los progresos del mundo de ficción se filtren en el mundo real a través de la mente del lector. Introduce la flexibilidad de lo que se considera real y, por consiguiente, abre la posibilidad de cambio. Es una literatura profundamente activista que invita al cuestionamiento y a la creatividad.

En el caso específico de Crónica del desamor, este activismo es obviamente feminista. Las personajes escriben para crear un nuevo espacio femenino en el discurso nacional. El 
acto de escribir les da a las mujeres de la novela la subjetividad que el patriarcado les ha robado. Observan el entorno social que las oprime, lo revelan y, con este descubrimiento de la imposición del silencio, lo resisten activamente. Nota Bárcenas Bautista (2007, p. 105) que

[...] es por medio del lenguaje que se puede llegar a nuevas formas de expresión femenina. El 'libro de las Anas' es el libro en el que aboga por una verdadera liberación femenina, en el que se deben romper silencios y tabúes impuestos para lograr la emancipación.

Crónica del desamor presenta la respuesta a la falta de voz de la mujer: la escritura. De modo metafictivo, las acciones de los personajes de la novela reflejan a la escritora real. Las mujeres de la novela, tal como lo hace Montero, usan su escritura para "romper silencios" impuestos por el patriarcado y liberarse de las restricciones y la subyugación implícitas en estos silencios. Shirley Montero Rodríguez (2006, p. 49) comenta sobre la escritora femenina y la construcción de la identidad:

Las mujeres de Crónica del desamor reconocen su condición social pasada y presente, como un primer paso para reencontrarse ellas mismas. No subvierten sólo exponen y refutan, desarrollando un verdadero discurso especular. Ya no son personajes atrapados al otro lado del espejo, sino que usan ese juego de especulación y especularización, de mirarse a sí mismas para mirar el entorno.

La escritura femenina, como la presenta Montero, les da a las mujeres de la novela la subjetividad que les ha robado tradicionalmente el patriarcado. Observan el entorno social que les oprimen, lo revelan, y con este descubrimiento del silencio impuesto lo resisten activamente.

Para lograr esta meta, las personajes de la novela escriben con dos propósitos distintos. El primero es revelar la verdad sobre la vida que llevan, las realidades ocultas y negativas de la Transición y la liberación falsa que han experimentado. Como profesora, Elena se acerca a este tema de manera científica; escribe un ensayo llamado "Pares e impares", "[...] un trabajo sobre los roles sociales, sobre un mundo hecho de estereotipos, de casilleros contrapuestos. Sobre la dificultad de ser impar y diferente, de escapar al papel tradicional o al par opuesto" (Montero, 1979, p. 99). Como una obra científica-sociológica, su ensayo se enfoca en evidencia y las conclusiones lógicas que sugiere. Se revela la verdad de una manera irrefutable, algo que legitima el descontento femenino. Irónicamente, esta forma de escritura científica, como la crónica discutida anteriormente, tradicionalmente le ha pertenecido al escritor masculino. La misma Ilustración, el movimiento filosófico que hizo popular la investigación científica, excluía la voz femenina. Como nota Mercedes Carbayo-Abengózar (2000, p. 111):

\footnotetext{
The Enlightenment was a project that excluded women; since the subject, the reason and the logos were all masculine. Feminism appeared as a reaction to this exclusion. During the twentieth century, women fought to be included in this masculine project.
}

En "Pares e impares", Elena subvierte las expectativas sociales, usando la misma forma de pensar y escribir que los ilustrados le negaban. Adopta el estilo de "poner más datos", como Mateo le pide a Ana que haga, pero se niega de "dejar que las conclusiones las saque el lector". Toma la autoridad completa de la voz científica masculina de la Ilustración y la emplea para explorar, cuestionar, y cambiar la situación de la mujer española. Bárcenas Bautista (2007, pp. 75-76) conecta este tipo de apertura social con la revelación de la experiencia sexual de Elena también:

\footnotetext{
Elena rompe el silencio sobre el sexo oral con el fin de que sus amigas se sientan identificadas con esta experiencia. El vívido lenguaje femenino utilizado en el recuento de esta historia deja la puerta abierta para que otras mujeres puedan liberar sus voces y así ganar carrera contra la represión sufrida.
} 
Elena logra su meta de vincular a las otras mujeres con la experiencia del silencio sexual. Ana cita el ensayo de Elena cuando habla de su propia insatisfacción romántica:

\begin{abstract}
Piensa Ana que algo va mal, muy mal en todo. Que ella es en esto víctima, pero también cómplice. Que sigue fingiendo, temerosa de no dar la talla impuesta por una sexualidad machista que esclaviza hoy a hombres y mujeres. Temerosa de decepcionar en su imagen de amante prototípico, contribuyendo así a que el teatro se repita. Tan encadenados estamos a nuestro rol, en esta sociedad en la que vivimos a través de estereotipos. Come dice Elena en su ensayo de "Pares e Impares": hay un rol de hombre, otro de mujer. (Montero, 1979, p. 231)
\end{abstract}

"Pares e impares" sirve su propósito central: revela los estereotipos genéricos y deja que Ana también empiece a observar evidencia en su propia vida, para luego compartir la experiencia con otras. Así acaban de ser las "prisioneras [...] de su papel de amantes" (Montero, 1979, pp. 229-230) que han sido anteriormente. Al romper el silencio, se rompe también el estancamiento social que anteriormente ha definido las relaciones sexuales en España.

Elena no es la única escritora en Crónica del desamor. En varios momentos de la novela, Ana piensa en la novela que luego escribe:

\begin{abstract}
Piensa Ana que estaría bien escribir un día algo. Sobre la vida de cada día, claro está. Sobre Juan y ella. Sobre Curro y ella. Sobre la Pulga y Elena. Sobre Ana María, que ha perdido el tren en alguna estación y ahora se consume calladamente en la agonía de saberse vieja e incapaz de. Sobre Julita, muñeca rota tras separarse del marido. Sobre manos babosas, platos para lavar, reducciones de plantilla, orgasmos fingidos, llamadas de teléfono que nunca llegan, paternalismos laborales, diafragmas, caricaturas y ansiedades. Sería el libro de las Anas, de todas y ella misma, tan distinta y tan una. (Montero, 1979, p. 8)
\end{abstract}

Esto es el elemento central de metaficción de la novela; Montero incluye unos momentos de esta narración:

Presentí que en sus palabras se encerraba una especie de exigencia, como si esperase un comportamiento
que yo ignoraba y que por unos momentos busqué desesperadamente. Para ganar tiempo, intenté esconder
mi cara con una expresión meticulosamente neutra: necesitaba mucho ese trabajo. (Montero, 1979, p. 137)

Como Elena, Ana escribe para documentar la realidad. Además, como Elena, emplea un género literario tradicionalmente dominado por la voz masculina: su "libro de las Anas" es una crónica, un relato sobre la realidad - en este caso, hechos normales, cotidianos y universales a todas las mujeres. Las personajes que Ana pone en la lista, tal como "las Anas" que reconoce, son todas únicas pero unidas en su experiencia de opresión. A través del relato de Ana, comparten sus experiencias no solamente con los otros personajes femeninos, sino con el lector también. Por sus aspectos de metaficción, esta obra borra las fronteras entre la realidad y la ficción y obliga que el lector reconozca que es un relato experiencial, no solamente una novela apartada de la realidad.

El segundo propósito que lleva a las personajes a escribir es para efectuar cambios. Ana empieza a escribir con el propósito de revelar la realidad, pero la trayectoria de esta meta cambia a través de la novela. Cuando Ana concibe "el libro de las Anas", solamente trata de la experiencia de la mujer española dentro de su entorno de represión. Según Montero Rodríguez (2006, p. 51),

Es en principio una escritura desde lo privado, lo rutinario, lo cotidiano, lo doméstico: sus amigos, problemas, inquietudes y afectos. Es decir, desde el encierro femenino, ensimismamente obligatorio a causa de una estructura que la aliena a ello [...] Es una escritura liberadora pero amarga.

Este primer modelo de escritura nace y existe dentro del sistema patriarcal, pero crea un espacio para otro tipo de expresión que mueve el enfoque desde la experiencia interior hacia el activismo textual-llega a construir encima de esta base experiencial una modalidad 
cambiadora: crea el "yo" femenino, un espacio para la mujer que trasciende y que se construye independiente del patriarcado, o, como lo describe Montero Rodríguez (2006, p. 52), “[...] hacia la búsqueda interna de su propio 'yo' (no el impuesto por el patriarcado), y externa, de su lugar dentro de la estructura social”. Según Bárcenas Bautista (2007, p. 77), Montero imbuye la escritura en su novela con dos metas esenciales: "dar testimonio" y "despertar consciencia". Crónica del desamor es simultáneamente una obra que revela la realidad y una obra que lucha activamente contra la opresión. Montero Rodríguez (2006, p. 45) propone que esta función liberadora se basa mayoritariamente en la idea de la escritora femenina: "La autora se autoafirma, dice ‘yo soy', 'yo quiero' y ‘yo deseo'; y esto es prohibido, por lo que se le descalcifica con denominaciones como de loca, irracional, esquizofrénica”. El acto de escribir es una afirmación de capacidad y subjetividad: requiere, implica y crea independencia del modelo patriarcal que ha oprimido a las mujeres. Las personajes-escritoras de la novela de Montero, por lo tanto, "assume their subjectivity as authors of their own life narratives" (Knights, 1999, p. 84).

Ana se apropia de su subjetividad al final de la novela, una acción definitiva que coincide con un cambio en su escritura. Por fin consigue acostarse con Eduardo Soto Amón, el objeto de una obsesión prolongada, y presiente cómo terminará la interacción: el hombre llamará un taxi para deshacerse de ella, "Y yo me sentiré ridícula, defraudada, y le diré que no, que no me importa..." (Montero, 1979, p. 258). Reconoce en esta situación el mismo silenciamiento de la mujer: algo le pasará que no le gustará, pero tendrá que suprimir sus propias opiniones y deseos para complacer al hombre. De repente, Ana opta por cambiar la situación: le dice a él que ella misma va a llamar un taxi. Inmediatamente, invierte la situación normal de demanda masculina y silencio sumiso femenino:

\footnotetext{
"[S]eguro", contesta Ana. Eduardo la contempla en silencio unos segundos, se le ve incómodo, titubeante. Al fin dice, “oye, ¿estás bien?", y envuelve el acento ansioso de sus palabras con una pátina de paternalismo. Ana siente súbitamente unos histéricos, irrefrenables deseos de reír, "yo estoy muy bien, mejor que nunca, ¿y tú?”, responde con entrecortadas carcajadas. Después le besa levemente en la mejilla, da media vuelta, se aleja por la solitaria calle, atrás queda Soto Amón, inmóvil sobre la acera, mirándola. (Montero, 1979, p. 259)
}

Ana se libera de la norma machista y rechaza el paternalismo con que Soto Amón pretende reestablecer la jerarquía sexual. Ana afirma sus opiniones, emociones e independencia de acción, y ahora es Soto Amón el que se calla. En este momento de inversión se cambia el propósito de la escritura de Ana. Opina,

\footnotetext{
[...] sólo le duele que fuera el propio Soto Amón quien se quitara la corbata en un automático, bien ensayado, autosuficiente gesto. Un gesto cruel y poderoso que, quién sabe, recapacita ella con ácida sonrisa, puede ser un buen comienzo para ese libro que ahora está segura de escribir, que ya no será el rencoroso libro de las Anas, sino apunte, una crónica del desamor cotidiano, rubricada por la mediocridad de ese nudo de seda deshecho por la rutina y el tedio. (Montero, 1979, p. 260)
}

La corbata de los hombres de negocios, los políticos y los burócratas, como símbolo masculino y fálico, representa el poder del patriarcado en el país. Del gesto "cruel”, "poderoso", y "autosuficiente" de quitarla, una representación visual del control del patriarcado, surge la imagen principal de la novela de Ana; es una imagen de poder insensato e injusto. Llega a ser no solamente un libro que revela las frustraciones de las mujeres españolas, sino también una obra que cuestiona, ataca, y desestabiliza el patriarcado español.

Crónica de desamor analiza la sociedad española de la Transición democrática desde un punto de vista feminista. Así lo interpreta Catherine Davies (1994, p. 97): 


\begin{abstract}
The female protagonists, in their thirties and forties, find themselves in situations which not only force them to reconsider traditional ideas and values regarding women's role in society, but also to seriously question the apparently more progressive solutions resulting from feminist debate, the Women's Liberation Movement, and the sexual revolution. The women in the novel must assess and reshape their lifestyles [...]. Above all, they need to find a new identity, a meaning and a purpose in life.
\end{abstract}

La cultura dominante de los setenta, un residuo del franquismo, oprime activamente a la mujer; el movimiento de liberación de la mujer, que les prometió a las españolas igualdad, solamente creó una desigualdad nueva y más abiertamente sexualizada. En el trabajo como en la cama, en la casa como en la calle, las mujeres de la novela se encuentran silenciadas en una sociedad dominada por los hombres -como Margarita, quien se cortó la lengua en el manicomio, no pueden expresarse. Sin embargo, a través de las acciones de sus personajes, Montero ofrece la oportunidad de "assess and reshape" la experiencia femenina española, como ha escrito Davies (1994, p. 97). Las escritoras-personajes de Crónica del desamor anuncian la injusticia callada de sus vidas y, a través del acto de escribir, toman subjetividad de sus experiencias. Cambian, entonces, la cultura de sumisión y silencio femeninos y encuentran un espacio, en este caso literario, en que pueden, por fin, hablar.

\title{
Bibliografía
}

Alborg, C. (1988). Metaficción y feminismo en Rosa Montero. Revista de Estudios Hispánicos. 1 (22), 67-76.

Bárcenas-Bautista, C. (1991). La mujer española y la deconstrucción del discurso misógino en Crónica del desamor, La función delta, y Te trataré como una reina de Rosa Montero. (Tesis Doctoral). University of Houston.

Brown, J. L. (1991). Women Writers of Spain: An Historical Perspective. Por J. L. Brown. (Ed.). Women Writers of Contemporary Spain: Exiles in the Homeland. (13-25). London: Associated University Presses.

Carbayo-Abengózar, M. (2000). Feminism in Spain: A History of Love and Hate. Por L. Twomey. (Ed.). Women in Contemporary Culture: Roles and Identities in France and Spain. (111-126). Portland: Intellect Books.

Davies, C. (1994). Contemporary Feminist Fiction in Spain. Oxford: Berg Publishers Ltd.

Escudero, J. (2006). Crónica del desamor, de Rosa Montero: un proyecto narrativo en ciernes. Por C. Prieto. (Ed.). No más sexo débil: la escritura española en el nuevo milenio. (35-54). Alicante: Universidad de Alicante.

Hooper, J. (1995). The New Spaniards. London: Penguin.

Jasper, A. D. (2005). Humor and Irony in the Postwar Writing of Carmen Martin Gaite, Rosa Montero and Carme Riera: 1978-1988. (Tesis doctoral). University of Michigan.

Knights, V. (1999). The Search for Identity in the Narrative of Rosa Montero. Lewiston: The Edwin Mellen Press.

Montero-Rodríguez, S. (2006). La autora femenina y la construcción de la identidad en Crónica del desamor de Rosa Montero. Revista de Filología y Lingüística de la Universidad de Costa Rica. 32 (2), 41-54. 
Montero, R. (1979). Crónica del desamor. Madrid: Debate.

Montero, R. (1999). Hace Treinta Años. Crónica del desamor. (2 ed.). Madrid: Debate. 
